\title{
Les autobiographies des cheminots italiens
}

Autobiographies of Italian railwaymen

\section{Stefano Maggi}

\section{OpenEdition}

\section{Journals}

Édition électronique

URL : https://journals.openedition.org/rhcf/1614

DOl : 10.4000/rhcf.1614

Éditeur

Rails \& histoire

Édition imprimée

Date de publication : 10 février 2013

Pagination : $57-70$

ISSN : 0996-9403

\section{Référence électronique}

Stefano Maggi, "Les autobiographies des cheminots italiens ", Revue d'histoire des chemins de fer [En ligne], 44 | 2013, mis en ligne le 13 novembre 2014, consulté le 22 avril 2022. URL : http:// journals.openedition.org/rhcf/1614; DOI : https://doi.org/10.4000/rhcf.1614 
Stefano MAGGI

\section{Les autobiographies des cheminots italiens}

auteur de ce panorama est le petit-fils d'un cheminot, le fils d'un cheminot et fut cheminot lui-même de 1987 à 1990. Ce n'est qu'en 2004 que le dernier oncle cheminot prit sa retraite, mettant un terme à une tradition familiale qui peut éclairer la lecture de ce qui va suivre*.

En Italie, l'autobiographie a d'abord été un genre pratiqué surtout par des hommes politiques puis, au $\mathrm{Xx}^{\mathrm{e}}$ siècle, par les combattants des deux guerres mondiales qui ont multiplié les témoignages. Les progrès de l'alphabétisation ont en effet engagé de nombreux sujets à se faire auteurs et écrivains de leur propre expérience, en particulier des périodes de leur vie, et les guerres le sont au premier chef, qui les ont obligés à sortir d'une routine quotidienne.

L'écriture des souvenirs de la vie professionnelle et de la vie au travail est beaucoup moins fréquente; cependant, le nombre des cheminots - qui ont été, pendant un siècle entier, des années 1870 aux années 1980, le groupe professionnel le plus important en Italie - explique l'existence d'un corpus de souvenirs de cheminots touchant à cette période. Plus encore, leur nombre a favorisé dans la deuxième moitié du siècle dernier la publication de mémoires

\footnotetext{
* Traduit de l'italien par Walter Gennari, avec la collaboration de Marie-Noëlle Polino.
} 
de fils (plus souvent que de filles) de cheminots qui, parvenus eux-mêmes à la maturité, ont raconté les événements de leur enfance qui impliquent les trains, la vie dans les gares ou dans les maisons de cantonnier. Or ces auteurs " secondaires " sont habitués à l'écriture et, s'ils ont grandi dans le monde des chemins de fer, ont eu également l'expérience d'autres milieux et viennent du journalisme, de la télévision ou du théâtre. Leurs souvenirs n'en sont que plus intéressants, car ces mémoires montrent comment l'environnement ferroviaire a influencé leur formation et leur vie ultérieure et comment l'appartenance à la "grande famille du rail ", avec ses caractéristiques sociales, ses savoirs techniques, ses aspects bureaucratiques, a été intériorisée par des générations successives. En effet, la reproduction sociale et professionnelle a été l'un des traits les plus marquants de la communauté cheminote et tout au long du $\mathrm{xx}^{\mathrm{e}}$ siècle, une génération après l'autre, les enfants des cheminots ont exercé la même profession que leur père et parfois fait le même travail.

Le rapide changement technique, avec la « fin du chemin de fer à l'ancienne " et la restructuration massive qu'a connue le secteur dans les vingt dernières années, pendant lesquelles le nombre des agents des chemins de fer a diminué au moins de moitié, a amené des retraités (souvent encore jeunes) à réfléchir sur leur expérience de vie, qu'ils ont souvent parée de la nostalgie d'un passé fait d'amitiés irremplaçables qu'offrait alors la "grande famille du rail » idéalement unifiée.

Les autobiographies que nous présentons ici constituent un échantillon d'un corpus plus important qui n'a pas encore été complètement défini. En effet, les textes qui traitent du monde ferroviaire en référence à des expériences vécues sont très nombreux et de plus en plus fréquents dans la presse ferroviaire et les magazines d'amateurs.

\section{Autobiographie syndicale}

Le genre autobiographique, dans le monde cheminot italien, est né dans la deuxième moitié du XIx ${ }^{e}$ siècle, avec les mémoires de dirigeants syndicaux et l'expression des plaintes du personnel ferroviaire exploité par les compagnies privées.

Un des premiers textes publiés a été en 1889 celui de Tito Livio De Dominicis, Vent'anni in ferrovia, écrit pendant sa vie active et publié après une retraite que la maladie avait anticipée. Né en 1851, De Dominicis a été embauché au chemin de fer en 1869, à la Société des chemins de fer du Sud (Società per le Strade Ferrate Meridionali). Il était télégraphiste et pendant sa période d'essai de six mois pouvait être envoyé vers n'importe quelle 
destination. Appelé au service militaire en 1871, il quitta l'armée en 1874 et revint au service des Chemins de fer du Sud, dans la région des Pouilles, à la gare d'Ortanova.

Ortanova est une ville où règne le paludisme, et le village lui aussi, qui compte environ 4000 habitants, n'en est pas du tout exempt. La gare est à $14 \mathrm{~km}$ de Foggia et à environ $13 \mathrm{~km}$ du village, de sorte qu' elle se trouve en pleine campagne et est horrible. De tous côtés, elle est entourée par des marais qui rendent l'air très malsain en été, et ainsi le personnel est contraint à prendre chaque jour de la quinine pour empêcher les crises pernicieuses qui sont fréquentes dans ces lieux. Environ deux mois après mon arrivée à Ortanova, je subis une attaque violente de fièvre qui me rendit méconnaissable, en dépit de ma bonne santé ${ }^{1}$.

Sa description de la vie ferroviaire est absolument négative : "Pour le personnel des chemins de fer, il n'y a ni loi ni règlement, tout dépend des caprices des supérieurs. La compétence n'est pas une raison d'espérer, l'honnêteté est une inaptitude, seuls ceux qui volent ou espionnent peuvent s'attendre au bonheur et à la bienveillance ${ }^{2}$. " Il critiquait aussi la gestion bureaucratique, caractérisée par « un grand fatras d'ordres de service qui le plus souvent ne mènent à rien, obscurs, incertains [...] et souvent copiés d'autres administrations $"^{3}$.

Le livre de De Dominicis fut suivi de celui d'Arturo Catelani, Vita ferroviaria. Catelani, après sa retraite des chemins de fer, devint écrivain et journaliste politique, d'orientation républicaine. Ses mémoires qui paraissent en 1894 sont une accusation directe contre les compagnies de chemin de fer et contre la gestion privée. Il évoque ainsi, à propos des accidents de chemin de fer, la commission d'enquête qui était nommée après chaque accident :

Après d'innombrables réunions et jetons de présence, après de longs procès-verbaux et ce que cela coûte en secrétariat, après une perte de temps, de papier et d'encre à ne pas y croire, tout cela se termine [...] avec la mutation d'office ou la rétrogradation d'un commis ou d'un chef de gare, avec le licenciement d'un aiguilleur ou d'un porteur qui, tous, sont autant responsables qu'un cheval de trait l'est de la rupture d'un chargement de meubles empilés au hasard sur une charrette que la gravité précipite à terré ${ }^{4}$.

Remarquables et très importants pour l'historiographie sont les textes de Cesare Pozzo, président de la Société de secours mutuels des mécaniciens et des chauffeurs (aujourd'hui Société nationale de secours mutuel Cesare Pozzo) qui, au cours des années 1880 et 1890, a écrit une série de réflexions sur la vie

1- Tito Livio De Dominicis, 20 anni in ferrovia, Naples, 1889, p. 18.

2-Ibid., p. 13.

3- Ibid., p. 10-11.

4- Arturo Catelani, Vita ferroviaria, Rome, Tip. dell'Unione cooperativa editrice, 1894, p. 13. 
des agents de conduite 5 couronnée en 1899 par l'œuvre posthume Vent'anni di vita ferroviaria. Storia dell'organizzazione dei macchinisti e fuochisti italiani.

Dans ce livre, publié un an après sa mort tragique - il se suicide en mai 1898 -, Pozzo décrit, à la troisième personne, la difficile évolution de l'organisation des cheminots vers le secours mutuel et le syndicalisme. Intéressante aussi fut sa participation aux assemblées générales des compagnies ferroviaires en qualité de représentant des travailleurs, que son ouvrage évoque ainsi :

Monsieur Pozzo, vu que les Actionnaires et le Personnel du chemin de fer représentent deux classes sociales continuellement en lutte entre elles, à cause de leurs intérêts et aspirations respectifs qui sont opposés, admet comme actionnaire - la bonne foi des deux parties en conflit et revendique, pour lui-même et pour ses camarades, représentants avec lui le personnel des chemins de fer et les catégories intermédiaires et inférieures, un traitement humain, même s'il dira des choses qui ne plaisent pas au président qui dirige la séance ${ }^{6}$.

Les ouvrages d'Ezio Gallori, Storie e racconti di un macchinista del Novecento et Quaranta anni di lotte in ferrovia da sindacato a Cobas, ont perpétué au $\mathrm{Xx}^{\mathrm{e}}$ siècle la tradition des témoignages syndicaux. Gallori décrit en détail non seulement les étapes du développement de l'action syndicale autonome des mécaniciens mais aussi plusieurs épisodes de sa vie professionnelle :

À la fin de ma longue et intense vie ferroviaire, qui a duré environ quarante ans, j'ai trouvé utile d'écrire ce livre, non seulement pour raconter "les mille et une histoires de grand-père" de ceux qui ont exercé avec tant de passion et de zèle ce métier dynamique et extraordinaire qu’est le métier de mécanicien, mais surtout afin que puisse rester le souvenir d'itinéraires et de luttes syndicales dont j'ai été un animateur avec beaucoup d'autres.

Donc, un livre de souvenirs professionnels, avec des personnages, des faits historiques, mais aussi des épisodes curieux et dramatiques (déraillements, tamponnement des heurtoirs, franchissement de sémaphores, télescopages de locomotives et de trains, erreurs qui auraient causé des tragédies corrigées à la dernière minute, inondations, trains à vapeur, trains de secours, etc.) qui sont plus ou moins arrivés à tous les mécaniciens qui comme moi ont parcouru deux millions de kilomètres sur les voies, mais aussi un livre qui veut être utile à ceux qui continueront, avec la passion qui a été la mienne, à se battre pour défendre et améliorer les conditions de vie et de travail des mécaniciens, des cheminots et de tous les travailleurs?

5- Voir par exemple, Cesare Pozzo, Scritti varii, Milan, Tipografia degli Operai (Soc. Coop.), 1889. À propos de la figure de Cesare Pozzo : Stefano Maggr, Il tormento di un'idea. Vita e opera di Cesare Pozzo : dal sindacato al socialismo (1853-1898), Milan, Angeli, 1998.

6- Cesare Pozzo, Vent'anni di vita ferroviaria. Storia dell'organizzazione dei macchinisti e fuochisti italiani, Milan, Koschitz, 1899, p. 136.

7- Ezio Gallori, Quaranta anni di lotte in ferrovia. Da sindacato a Cobas, Florence, Ancora In Marcia, 1996, p. 1. 
Résumant son expérience de mécanicien, Gallori écrit :

J'ai commencé à travailler aux chemins de fer en 1957, vu que je me classai 14 è un concours d' "élève assistant mécanicien extraordinaire à l'essai" que mon oncle Roger, ancien chef-technicien FS, me conseilla de tenter. Je venais d'un typique "petit village de campagne" où l'unique activité culturelle était celle d'un prêtre auprès duquel, nous, les enfants, étions enfants de chœur.

J'affrontai neuf mois d'école professionnelle d'assistant mécanicien, je passai l'examen final avec une note de 115/120, score qui m'empêcha d'émigrer de Florence d'où je ne m'éloignais plus. L'école a été une période d'étude intense, où tout le monde (et surtout moi) " potassait ", une école qui a produit de bons mécaniciens, beaucoup de chefs de dépôt, et même des dirigeants des FS et surtout beaucoup de syndicalistes de tous les syndicats [...]

Je fus intégré dans le classement du tableau fourni du personnel roulant de Florence ( $221^{\mathrm{e}}$ parmi les assistants mécaniciens disponibles) puis, après le stage pratique, suivis une longue progression : chargé du wagon réchauffeur à vapeur, de la manœuvre de locomotives à vapeur, des trains supplémentaires, avant d'affronter les examens de mécanicien (1963) auxquels on ne pouvait se présenter qu'à 25 ans. Et ainsi de suite, avec les trains à vapeur, avec les nombreux certificats d'aptitude à la conduite à obtenir pour les nouvelles locomotives, et les examens médicaux périodiques à passer.

Un long voyage fait avec des « maîtres » et de nombreux collègues avec lesquels j'ai partagé le pain quotidien, la vie de tous les jours, et avec beaucoup d'entre eux je me sentais et je me sens encore lié comme à des frères...

Ce long voyage se termina le 7 février 1996, à l'âge de 58 ans, date prévue pour la retraite des mécaniciens, alors que cela faisait une année que j'occupais le premier rang dans le classement à l'ancienneté de service des mécaniciens du dépôt des locomotives de Florence ${ }^{8}$.

C'est en tant que cheminots protagonistes des événements de leur temps que Cesare Pozzo, pendant le dernier quart du XIx $x^{e}$ siècle, et Ezio Gallori, au siècle suivant, ont écrit leurs mémoires, partageant la forte conviction d'avoir joué un rôle clé dans le groupe professionnel des mécaniciens et d'avoir affirmé et consolidé l'identité de ce métier.

\section{Le témoignage des écrivains}

L'histoire de Cesare Pozzo, sans doute le cheminot italien le plus connu, souvent mentionné dans l'historiographie, est emblématique de la syndicalisation et de la politisation des cheminots, une adhésion au socialisme si forte et si claire dans les premières années $\mathrm{du} \mathrm{Xx}^{\mathrm{e}}$ siècle qu'elle est évoquée dans des œuvres littéraires qui ne sont pas liées aux chemins de fer. Par exemple, le ro-

8-Ibid., p. 4. 
man d'Ugo Ojetti, Mio figlio ferroviere : romanzo (paru en 1922), dans lequel à propos d'un médecin et de son fils Nestor, l'écrivain met en scène le lien entre le métier de cheminot et le socialisme, après la Première Guerre mondiale :

Au printemps de 1919, Nestor rentra à la maison, avec son congé de démobilisation. Je le vois comme s'il était devant moi maintenant. Il était vêtu de serge bleue, avec une chemise habillée de laine noire et un papillon noir.

- Je dois partir à nouveau après-demain, m'annonça-t-il quand je l'embrassai et le serrai dans mes bras.

- Pour aller où ?

- À Turin, à l'école des mécaniciens.

- Quels mécaniciens?

- Mon père, j’ai choisi mon métier. Je deviendrai cheminot.

- Quoi ?

Et il prit, du pot de fleurs qui se trouvait au milieu de la table dressée, un œillet rouge, et le mit à sa boutonnière?.

Au même moment se situe la publication du livre Ricordi di un impiegato de Federigo Tozzi, un écrivain né en 1883 qui fut brièvement cheminot et dont les souvenirs concernent le travail en gare.

C'est en 1908 qu'il réussit un concours d'entrée aux chemins de fer et commença à travailler en Toscane, à la gare de Pontedera où il resta deux mois. Il fut ensuite muté à Florence où il ne resta que trois semaines avant de démissionner à la mort de son père qui dirigeait une auberge.

Ces "souvenirs d'un employé ", probablement écrits en 1910, forment un roman par lettres issu de la correspondance de Tozzi avec sa future épouse. On en retient l'image très forte de l'agent qui, enveloppé dans une solitude qui l'« étourdit » et l'" assourdit ", face à l'« indistinct fourmillement » venant de la plaine, interroge les voies sans comprendre ce qu'elles veulent lui dire. Puis, soudain, ses craintes se concrétisent : un train express (direttissimo) passe à toute allure. Ce détail le met en présence de la possibilité de la mort : " Je sais qu'il renversera un garde voie, en lui emportant la tête au crochet d'une lanterne ${ }^{10} . »$

La situation de cheminot, sûre et bien payée, était l'ambition de la petite et moyenne bourgeoisie. Tozzi, qui se considérait comme un rebelle, ressent avec vigueur la contradiction entre cet état et le tourment de son âme

9- Ugo Ojeтtr, Mio figlio ferroviere : romanzo, Milan, Treves Editore, 1922.

10- Federigo Tozzi, Con gli occbi cbiusi : Ricordi di un impiegato, Milan, Feltrinelli, 1994, p. 144. 
d'artiste : « La gare, où je dois rester à la chaîne comme un chien dans sa niche en bois, est petite, mais la campagne s'étend librement. N'est-il pas possible qu'un jour je m'en aille ${ }^{11}$ ?"

Pour ce qui est de la représentation du réel, il brosse certains tableaux de la vie ferroviaire, comme le moment de la mise en ordre des documents de service à la fin de la journée, la place de la gare avec le va-et-vient des charrettes, l'arrivée du tramway, les repas pris au buffet.

Semblable par sa construction à un roman autobiographique, bien que très différente par son style, l'œuvre de Piero Jahier, Resultanze in merito alla vita e al carattere di Gino Bianchi, paraît en 1915. Jahier, licencié en droit, était inspecteur des chemins de fer. Dans ce roman, il décrit la vie d'un employé de l'administration qui identifie sa vie avec la durée de son service. Dans le texte apparaissent des expériences autobiographiques, par exemple quand est décrite la cadence de travail de l'administration publique, à laquelle appartenaient les chemins de fer depuis la nationalisation de 1905. Le protagoniste Gino Bianchi est commissionné par la Société des chemins de fer du Sud en 1893, puis déplacé aux Chemins de fer de l'État. Jahier décrit la période d'agitation qui a précédé la création de l'entreprise d'État, qu'il avait vécue directement.

Après la tempête et l'agitation, le jour de la réforme inévitable des chemins de fer arriva.

Les agitateurs avaient tout ébranlé, et les associations respectables, les consortiums avaient voté d'innombrables ordres du jour, le gouvernement, financé d'innombrables commissions et la presse, achetée, s'était salie et servait la politique.

C'est pendant ces jours-là, de fait, que certains rédacteurs en chef découvrirent ce qu'est un train : un garde-manger qui roule, menacé par les hommes, par le soleil et par le vent ${ }^{12}$.

D'évidence, le passage d'une gestion privée à la gestion publique des chemins de fer, qui a constitué dans l'histoire du $\mathrm{Xx}^{\mathrm{e}}$ siècle italien un véritable tournant, dans le contexte global de l'évolution des services publics, revêtit une signification importante pour les itinéraires individuels des contemporains : il suffit de penser à l'angoisse que peut générer le passage d'un employeur à un autre et à ce que cela a signifié pour la vie quotidienne des cheminots.

11- Ibid., p. 145.

12- Piero JAHIER, Resultanze in merito alla vita e al carattere di Gino Bianchi, con un allegato, Florence, Libreria della Voce, 1915, p. 125. 


\section{Textes autobiographiques et autres écrits professionnels}

Dans le dépôt de l'Archivio Diaristico Nazionale Pieve Santo Stefano (Arezzo), on trouve quinze textes autobiographiques liés aux cheminots et au contexte ferroviaire, dont certains dépeignent la vie professionnelle des cheminots. Le récit de Danilo Gracci, qui se réfère aux années 1927-1933, celui d'Enzo Losi, qui couvre toute sa vie (1911-2001), celui enfin de Mario Tarallo, qui traite les années de la Grande Guerre (1915-1918), sont particulièrement intéressants.

Gracci, qui était chef de gare, a laissé un récit complet, riche en noms et épisodes détaillés, qui va de la Sicile à la Toscane, où il décrit certains milieux ferroviaires de façon négative ${ }^{13}$. Malgré la conscience de la « modestie de [son] emploi ", la famille de deux enfants d'un chef de gare italien dans les années 1930 ne pouvait pas être appelée " prolétaire » comme celle d'autres ouvriers ${ }^{14}$. Elle dispose d'un logement fourni par les chemins de fer dans la gare. Gracci envoie son fils au lycée, il voyage avec lui de Pontedera à Florence, en Toscane, et transporte son vélo sur le train. En août 1932, il part en vacances avec sa famille en haut-Adige pendant trois semaines.

Ce récit révèle une vie de responsabilités, de privations à cause des mutations fréquentes, mais aussi de satisfactions du travail accompli, en dépit du poids de la hiérarchie, rigide, de l'administration des chemins de fer.

Dans les mémoires d'Enzo Losi figure une référence à la famille cheminote, avec quelques commentaires intéressants sur l'exode rural qui conduit de la campagne vers les chemins de fer. Il évoque également ses souvenirs de l'environnement ferroviaire dans lequel il a vécu depuis son enfance, étant le fils d'un cheminot obligé à de fréquents déménagements entre l'Émilie et la Lombardie ${ }^{15}$.

Le texte de Mario Tarallo (classe 1899, la dernière qui partit combattre lors du premier conflit mondial), recèle certains épisodes de la guerre mais, à partir de considérations familiales éparses, apparaissent des remarques significatives à

\footnotetext{
13- Danilo Gracci (1896-1984), récit (1933) écrit en 1927-1933, Archivio Diaristico Nazionale onlus (désormais ADNo), DP/94.

14- Danilo Gracci était le fils unique d'un paysan, lui-même le benjamin d'une fratrie de 15 enfants. Son père a été le premier à quitter la ferme à 18 ans parce qu'il s'était querellé avec le fermier à propos du contrat de fermage. Il alla en Maremme, s'enrôla comme carabinier, quitta l'armée pour se marier et ouvrit un bistrot qu'il dut quitter après quatre ans de mauvaises affaires. Il fut par la suite employé au bureau des taxes de l'octroi de la municipalité de Livourne. Le fils Danilo put étudier et trouver un emploi dans les chemins de fer à un niveau intermédiaire, celui de chef de gare.

15- Enzo Losi (1911-2003), récit (2002) écrit en 1996-2002, ADNo, MP/05.
} 
propos de la transmission de père en fils des compétences et savoirs professionnels. Son père travaillait à l'usine Miani Silvestri, où il dessinait moteurs et locomotives, mais il mourut quand Mario avait six ans. Deux frères furent embauchés en tant que dessinateurs par les Chemins de fer de l'État, vers 1912, dans la continuité du travail paternel ${ }^{16}$.

Enfin, d'un grand intérêt est le récent livre d'Aldo Leonini, conservé à Pieve Santo Stefano (disponible également sur l'Internet), intitulé La mia vita ferroviaria, 1959-1984. En partie en prose et en partie en vers, l'auteur y décrit sa vie sociale comme chef de train de 1959 à $1984^{17}$.

L'Archivio Diaristico Nazionale ne clôt pas la série des souvenirs écrits par des cheminots à propos d'eux-mêmes. En effet, le soudain changement intervenu dans le monde ferroviaire depuis 1990 a relancé la publication de Mémoires.

On peut mentionner Nino Donadi, Oggi come ieri. Il ferroviere, un roman dans lequel l'auteur donne en 1998 une suite au film Il ferroviere (1956) du metteur en scène Pietro Germi, chef-d'œuvre cinématographique, unique en Italie ${ }^{18}$. Le personnage principal en est Sandro, le fils cadet d'Andrea Marcocci, le protagoniste du film. C'est un roman passionnant qui décrit avec justesse le milieu des chemins de fer. La préface de l'ouvrage, cependant, exprime toute l'amertume de l'auteur, mécanicien qualifié, face à la privatisation des Chemins de fer de l'État et à tous les événements qui ont influencé son itinéraire personnel et professionnel.

Toutefois ce sont les chefs de gare qui ont le plus subi ces mutations professionnelles, comme on peut le lire dans l'ouvrage d'Alberto et Francesco Lorenzini, paru en 2006, C'erano i 'Berretti Rossi' in ferrovia. Ottanta anni di storia con gli occhi e i sentimenti di due generazioni di Capi Stazione. Ce livre fait revivre quatre-vingts ans d'histoire ferroviaire, à travers les vicissitudes humaines et professionnelles d'Alberto et Francesco, père et fils, tous deux chefs de gare. Ils se sont donnés à leur travail avec une passion qui a marqué leur vie. Le livre, écrit par le fils, Francesco, reprend les souvenirs ferroviaires composés en rime par son père Alberto. Né en 1902, celui-ci partit à la retraite en 1964 au grade de chef de gare titulaire, en ayant occupé la plupart de ses emplois sur la ligne Bologne-Pistoia-Porretta Terme, sur les Appennins entre Émilie

16- Mario TARAllo (1899-1985), récit (1983) écrit la même année, ADNo, MG/92.

17- Aldo LeOnini (1926-), récit (2010), La mia vita ferroviaria 1959-1984, ADNo, en ligne à l'adresse suivante : http://leoninialdoraccontipoesie.sitiwebs.com (consulté en 2012).

18- Nino Donadi, Oggi come ieri. Il ferroviere, Rome, s.éd., 1998. 
et Toscane. Albert, né en 1933, prit sa retraite en tant que chef de poste de commandement à la fin des années 1990.

Le livre récent de Gerardo Rossi, Trilli ferroviari. Ricordi di un ferroviere in pensione (1989-2007), paru en 2009, raconte l'histoire d'un voyage de vingt ans dont les étapes sont la photographie des sensations associées à des moments particuliers vécus en Romagne avec des équipes de travaux d'entretien des voies ${ }^{19}$.

\section{Les récits des enfants de cheminots}

La première des autobiographies écrites par un fils de cheminot qu'il faut évoquer est celle du dramaturge Emilio Caglieri, parue en 1977, Il trenino per Vallombrosa.

L'auteur y décrit à travers ses yeux d'enfant une jeunesse romancée vécue en Toscane, sur la ligne Sant'Ellero-Saltino Vallombrosa, offrant une analyse qui relève de l'histoire microsociale. Le récit se déroule sur un chemin de fer à voie étroite, l'un des premiers chemins de fer touristiques italiens. Le père de l'auteur y tient le rôle de chef de dépôt et de chef d'atelier, le protagoniste prend le train tous les jours pour aller à l'école. De la lecture du texte on peut tirer des observations intéressantes sur la vie du fils d'un cheminot et même sur la gestion d'une compagnie ferroviaire d'intérêt local. Ouverte en 1892, cette ligne a été fermée en 1924. Cinquante personnes environ y travaillaient, c'est-à-dire le personnel des trains, des gares intermédiaires et des voies, tous employés d'une entreprise privée qui avait construit la ligne et assurait son exploitation.

Pour réduire les coûts chacun faisait tout son possible et tenait à exercer des tâches diverses. Exemple de cette polyvalence, celle du chef de dépôt, père de l'auteur :

Tenir les livres de comptes et diriger la circulation des trains, insignifiante en hiver mais considérable en été pendant les périodes principales d'affluence des visiteurs, travailler en tant que mécanicien, rétablir d'éventuels dommages subis par le matériel roulant, de plus travailler à la forge, à l'établi, au tour, et exécuter les travaux les plus difficiles parce que le meilleur ouvrier c'est lui. Et cela ne suffit pas. Au premier étage du bâtiment de la gare il y a un bureau où il tient des registres différents concernant l'administration du personnel et de l'atelier de réparation ${ }^{20}$.

19- Gerardo Rossi, Trilli ferroviari. Ricordi di un ferroviere in pensione (1989-2007), Faenza, Il Ponte Vecchio, collection « Soldanellae », 2009.

20- Emilio CagLieri, Il trenino per Vallombrosa. Storia di una ferrovia e di un ferroviere a scartamento ridotto, Florence, Libreria SP 44, 1977, p. 90. 
Une autre œuvre récente qui rappelle le monde des chemins de fer dans le cours d'un récit est celle de Walter Gennari. Enfant d'une mère et d'un père cheminots, il a pour cette raison développé une véritable passion pour les trains, qu'ils soient réels ou en miniature. En ce qui concerne le modélisme ferroviaire, les fils de cheminots ont exprimé leur créativité, qui a assez fréquemment une source autobiographique, précisément parce qu'ils sont fils ou petits-fils de cheminots, avec un parent qui travaille ou travaillait aux chemins de fer et qui entraînait les enfants dans le monde des trains. Dans le chapitre "Vu de l'extérieur et de l'intérieur" du livre Il Silenzio è d'oro. Treno fuori, treno dentro, Gennari écrit en 2006 :

Je me souviens avec quelle passion je regardais les trains quand j'étais enfant : je semblais malade de trains. Aujourd'hui, presque rien n'a changé même si je ne suis plus un gamin, et pourtant, en m'arrêtant pour regarder çà et là parmi les étalages des petits marchés des maquettes, japerçois tant de gens de tous les âges qui cherchent des reliques à l'échelle 1/87 avec parcimonie, d'autres qui comme des guêpes folles se damnent pour trouver des pièces de rechange pour des trains encore plus petits. C'est un ferment d'énergie peut-être contenue par rapport à celle que manifestent les supporteurs de football ou de sport automobile, mais très vivant, et puissant comme une locomotive à vapeur en pleine vitesse $\mathrm{e}^{21}$.

Quand on s'attache aux véritables autobiographies de fils de cheminots, celles qui sont racontées à la première personne, on doit citer le livre de l'écrivain Franco Piccinelli C'era una volta il treno, le meilleur dans sa catégorie pour représenter les cheminots italiens, dont il décrit en détail la hiérarchie et livre des scènes de la vie quotidienne sur une ligne secondaire du Piémont, gérée par les Chemins de fer de l'État. Son texte, paru en 1996, démontre une connaissance très approfondie du monde ferroviaire liée à sa vie de fils de chef de gare.

Quand il s'agit de voyager, ma préférence est d'ordre "génétique", car je suis né dans une gare, au milieu d'une ligne à une seule voie qui va vers les collines, puis débouche en chatouillant ses côtés [...] une gare splendide comme en pourrait exister une dans les contes de fées où ces dernières arrivent à tout faire briller pendant les journées grises.

Peinte en vert. Deux petits jardins sur les côtés. Une petite fontaine qui ne cessait de verser l'eau sulfureuse [...] Une grande place derrière, toujours pleine de gens qui allaient, venaient, s'arrêtaient, bavardaient [...] Trois voies de passage devant, trois voies de garage sur le côté [...] Bien qu'il ne fît pas partie au sens strict de la gare, il y avait un hôtel-restaurant bien fréquenté avec une cave d'où on pouvait sentir le vin [...] auprès des installations ferroviaires, au-delà de la grille. Si mon père assistait à l'arrivée

21- Walter Gennari, Il silenzio è d'oro. Treno fuori, treno dentro, Trente, Editrice UniService, 2006, p. 67. 
de chaque train en face de son bureau, avec son bonnet rouge sur la tête, le restaurateur aussi recevait le train en face de la porte d'entrée de l'hôtel, avec son long tablier vert, comme dans une opérette viennoise ${ }^{22}$.

Après l'évocation du milieu ferroviaire, avec sa hiérarchie rigide, les caricatures des " chefs " mais aussi la richesse des relations personnelles entre les cheminots, Piccinelli décrit enfin la gare pendant les dernières années du $\mathrm{xx}^{\mathrm{e}}$ siècle, le livre se termine ainsi sur une pointe d'amertume, à cause $\mathrm{du}$ changement radical intervenu dans le monde ferroviaire à cette époque :

Mon père, chef de gare principal, après ton long chemin de vie, quel cafard notre installation ferroviaire, dépouillée de tout et de vous. Les trains arrivent, s'arrêtent, repartent. Ce sont des trains régionaux métropolitains.

Les voies ont disparu. Il n'y en a qu'une seule.

Il n'y a pas l'ombre d'un cheminot sur le sol, ou de guichets, ni leviers d'ouverture du disque ou de fermeture des barrières. Les bérets rouges ont disparu. Même les rosiers grimpants en forme d'arche sur les cinq portes de la gare ont capitulé, ils ne fleurissent plus ${ }^{23}$.

La description la plus attrayante d'une famille cheminote est celle d'Ugo Pirro, Figli di ferroviere, où la famille apparaît d'abord dans son environnement social.

La mienne est une famille de cheminots : mon père, mon grand-père, mes oncles paternels étaient cheminots, les sœurs de mon père épousèrent des cheminots, aussi de nombreux cousins ont été cheminots et autant de mes cousines se marièrent à des chefs de gare, chefs de train, chefs d'exploitation, contrôleurs ${ }^{24}$.

Le livre de Pirro, paru en 1999, est un véritable récit autobiographique dans lequel il détaille les événements familiaux et leur contexte politique et économique, notamment les déménagements continus qui forcent la famille à une constante adaptation, même à des régions éloignées les unes des autres comme la Campanie, les Marches ou la Lombardie. Né à Salerne, en Campanie, Ugo Pirro était le fils d'un chef de gare. Son grand-père maternel était un propriétaire de Battipaglia, où il fut le premier maire après que la ville fut séparée d'Eboli. Son père avait ensuite été muté à Naples puis à Segni Paliano, dans une zone paludéenne où il resta peu et retourna à Naples après un hiver. À ce moment-là, ils allèrent vivre dans les grandes habitations collectives qui à cette époque avaient commencé à être construites pour les cheminots. À la fin de l'année 1928, ils déménagèrent à Napoli Mergellina (une gare

22- Franco Picineldi, C'era una volta il treno, Turin, Società editrice internazionale, 1996, p. 3-10.

23- Ibid., p. 149.

24- Ugo Pirro, Figli di ferroviere, Palerme, Sellerio, 1999, p. 9. 
monumentale qui venait d'être inaugurée), parce que le père avait été nommé chef de gare de seconde classe.

Les avancements de carrière, les promotions n'étaient pas seulement déterminés par l'ancienneté de service. Chaque cheminot recevait chaque année un jugement. Qui était évalué comme "excellent ", au fil des années acquérait le droit à une promotion, une amélioration de sa situation financière. Mais aussi, comme dans la carrière militaire, les punitions, les jours de suspension, les amendes, les mutations dans l'intérêt du service pleuvaient. À Naples Mergellina, mon père n'aurait enfin plus de service de nuit à accomplir. Quand il était en service à Naples Centrale les services de nuit étaient hebdomadaires. Il rentrait à la maison à sept heures du matin, bouleversé et en colère, muet à cause du sommeil. Il ne parlait à personne ${ }^{25}$.

Puis son père prit son service dans les Marches, à San Benedetto del Tronto, et termina sa carrière à Lecco, en Lombardie.

Pendant la journée qui fut la dernière de sa vie comme cheminot, nous nous sommes réunis tous autour de lui dans la maison de la gare. Après quelques jours ensemble, nous quittâmes la gare de Lecco, nous chargeâmes les meubles de notre maison sur un wagon de marchandises dirigé à Battipaglia. En train, Renato et moi accompagnâmes nos parents dans leur vieille maison au village où maman était née et avait grandi, où elle avait connu et aimé notre père. Les oncles et les cousins cheminots n'étaient plus là. Aux chemins de fer restait mon frère Renato, mais pas pour longtemps : il préféra d'autres activités.

Désormais, chaque station nous appartient un peu, c'est là que nos souvenirs sont déposés, ils courent sur les voies, ils s'arrêtent dans les gares où nous avons grandi ${ }^{26}$.

La fin du livre de Pirro fait réfléchir au dernier caractère de la " société cheminote ", celui-là même dont nous sommes ici, en tant qu'auteur, l'illustration : la fin des familles ferroviaires qui ont caractérisé le $\mathrm{xx}^{\mathrm{e}}$ siècle et dont, en ce début de $\mathrm{XXI}^{\mathrm{e}}$ siècle, il ne reste plus que quelques exemples.

\section{Références bibliographiques}

\section{Autobiographies et récits publiés}

Caglieri Emilio, Il trenino per Vallombrosa. Storia di una ferrovia e di un ferroviere a scartamento ridotto, Florence, Libreria SP 44, 1977.

Catelani Arturo, Vita ferroviaria, Rome, Tip. dell'Unione cooperativa editrice, 1894.

25- Ibid., p. 56.

26- Ibid., p. 155-156. 
De Dominicis Tito Livio, 20 anni in ferrovia, Naples, 1889.

Gallori Ezio, Quaranta anni di lotte in ferrovia. Da sindacato a Cobas, Florence, Ancora In Marcia, 1996.

Gennari Walter, Il silenzio è d'oro. Treno fuori, treno dentro, Trente, Editrice UniService, 2006.

Lorenzini Alberto et Francesco, C'erano i "Berretti Rossi » in ferrovia. Ottanta anni di storia con gli occhi e i sentimenti di due generazioni di Capi Stazione, Bologne, Associazione D.L.F., 2006.

Picinelli Franco, C'era una volta il treno, Turin, Società editrice internazionale, 1996.

Pirro Ugo, Figli di ferroviere, Palerme, Sellerio, 1999.

Pozzo Cesare, Vent'anni di vita ferroviaria. Storia dell'organizzazione dei macchinisti e fuochisti italiani, Milan, Koschitz, 1899.

- Scritti varii, Milan, Tipografia degli Operai (Soc. Coop.), 1889.

Rossi Gerardo, Trilli ferroviari. Ricordi di un ferroviere in pensione (19892007), Faenza, Il Ponte Vecchio, collection «Soldanellae », 2009.

\section{Récits non publiés, Archivio Diaristico Nazionale onlus (Pieve Santo} Stefano) (ADNo)

Gracci Danilo (1896-1984), récit (1933) écrit en 1927-1933, ADNo, DP/94.

LEONINI Aldo (1926-), récit (2010) en ligne, La mia vita ferroviaria 19591984, ADNo, à l'adresse suivante : http://leoninialdoraccontipoesie.sitiwebs.com (consulté en 2012).

Losi Enzo (1911-2003), récit (2002) écrit en 1996-2002, ADNo, MP/05.

Tarallo Mario (1899-1985), récit de 1983 écrit en 1983, ADNo, MG/92.

\section{Cuvres de fiction}

Donadi Nino, Oggi come ieri. Il ferroviere, Rome, s. éd., 1998.

JaHIER Piero, Resultanze in merito alla vita e al carattere di Gino Bianchi, con un allegato, Florence, Libreria della Voce, 1915.

Ojetti Ugo, Mio figlio ferroviere : romanzo, Milan, Treves Editore, 1922.

Tozzi Federigo, Con gli occhi chiusi : Ricordi di un impiegato, Milan, Feltrinelli, 1994.

\section{Bibliographie}

MagGi Stefano, Il tormento di unidea. Vita e opera di Cesare Pozzo : dal sindacato al socialismo (1853-1898), Milan, Angeli, 1998. 